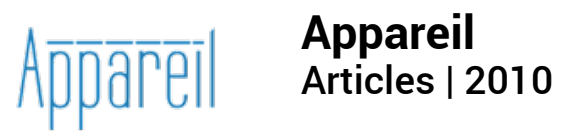

\title{
Le cimetière comme hétérotopie
}

\section{Alain Brossat}

\section{OpenEdition}

\section{Journals}

Édition électronique

URL : http://journals.openedition.org/appareil/1070

DOI : 10.4000/appareil. 1070

ISSN : 2101-0714

Éditeur

MSH Paris Nord

\section{Référence électronique}

Alain Brossat, "Le cimetière comme hétérotopie », Appareil [En ligne], Articles, mis en ligne le 29 septembre 2010, consulté le 30 juillet 2020. URL : http://journals.openedition.org/appareil/1070 ; DOI : https://doi.org/10.4000/appareil.1070

Ce document a été généré automatiquement le 30 juillet 2020.

\section{(c) (i) (9)}

Appareil est mis à disposition selon les termes de la Licence Creative Commons Attribution - Pas d'Utilisation Commerciale - Pas de Modification 4.0 International. 


\title{
Le cimetière comme hétérotopie
}

\author{
Alain Brossat
}

1 Évoquant, dans le texte intitulé "Les hétérotopies ", "une surdétermination de l'hétérotopie - les cimetières pour tuberculeux", Foucault écrit: «Je pense à ce merveilleux cimetière de Menton dans lequel ont été couchés les grands tuberculeux qui étaient venus, à la fin du XIX ${ }^{e}$ siècle, se reposer et mourir sur la Côte d'Azur : autre hétérotopie ».

2 Cette phrase m'a marqué, car il se trouve que ce cimetière m'est familier, lié à des souvenirs d'enfance, puisque j'ai passé, des années durant, les vacances de Pâques, avec mes parents, à Menton, sous la tente, au plateau Saint-Michel d'où l'on a une vue imprenable sur ce cimetière, et que j'ai eu l'occasion d'y faire un nouveau pèlerinage, tout récemment, puisque mes parents, maintenant âgés, se sont retirés dans cette ville vouée aux bains de mer et aux retraites cossues, jamais lassés de compter les vagues.

Assurément, en tout cas, appliquée à ce lieu particulier, la notion d'hétérotopie trouve un relief tout à fait saisissant. Le vieux cimetière de Menton est en fait constitué de deux isolats qui surplombent la vieille ville et le vieux port, la baie de Garavan et la frontière avec l'Italie. Le plus bas est celui dont parle Foucault, celui qui, en grande majorité, est "peuplé » de morts de la tuberculose, à la fin du XIXe, au début du Xx siècle. Le plus haut, dit du Rebuquet, est pour une part un cimetière militaire où sont enterrés des soldats, des coloniaux, morts des suites de blessures et autres affections contractées sur le front, pendant la première guerre mondiale, et, pour l'autre, des habitants du cru, appartenant à de vieilles familles mentonnaises.

Dans ces deux sites, la notion d'hétérotopie prend tout son sens. Ils constituent en effet, dans la ville dont ils font formellement partie, comme des espaces d'extraterritorialité : pour y accéder, il faut quitter la partie basse de la ville, bruyante, commerçante, parcourue pendant presque toute l'année par des hordes de touristes, avec son marché couleur locale, un peu trop couleur locale, ses rues piétonnes, monter des ruelles en pente raide, parcourir les venelles étroites de la vieille ville, avant d'arriver dans cet espace dont le propre n'est pas seulement d'être séparé (par les murs qui l'entourent, comme tout cimetière), mais surtout de produire comme une inversion de tous les signes courants de la vie urbaine: le silence y règne comme les rues, plus bas, sont 
vouées au vacarme de la circulation automobile, des chantiers, de l'agitation vacancière ; les vivants y sont rares et s'ils parlent, c'est à voix basse, leurs mouvements sont lents et mesurés comme ceux de la population d'«en-bas» sont fébriles, exubérants ou pressés, etc. Et pourtant, "l'autre ville», celle des vivants est tout proche, et cela se rappelle à nous par instants lorsqu'un coup de klaxon, un vrombissement de moto, des cris d'enfants jouant sur la placette devant l'église SaintMichel viennent trouer le silence de cet « espace autre » dans lequel, déjà, nous nous étions établis.

5 Le cimetière, bien sûr, c'est banal de le dire, est comme une autre « ville » dans la ville une ville peuplée de morts. Mais l'altérité prend ici, comme le relève Foucault, un sens radical: le rapport au temps s'y transforme totalement - c'est, dans la partie basse, davantage qu'un certain $x^{e}{ }^{e}$ siècle qui s'y trouve figé - quelque chose comme un "âge de la tuberculose » qui y est éternisé ou fossilisé. Et, un peu plus haut, au Rebuquet, un arrêt sur image, celle de la scène répétée à l'infini où les troupes coloniales sont envoyées à l'assaut des tranchées allemandes, en première ligne, et où ces poilus aux noms « indigènes » venus d'ailleurs (inscrits sur une pauvre plaque, sur chaque croix) tombent, et tombent et tombent sous le feu des mitrailleuses (Malgaches, Marocains, Sénégalais...). C'est ce monde " tout autre » où la mort, les morts qui, comme chacun sait, ont tout leur temps, imposent leur temporalité propre aux vivants, promeneurs, curieux, photographes et filmeurs, qui s'y aventurent. Une sorte de lenteur qui ralentit les gestes, fige les paroles, engourdit les émotions trop vives, s'empare du flâneur et le désoriente.

C'est aussi que, contrairement à ce qui pourrait être le cas dans le cimetière d'un village familier, tous ses repères se trouvent, en ce lieu, abolis : la société des morts qui s'y trouve rassemblée est, à tous égards, une anti-société : rassemblée non pas par les lignages, les alliances, les systèmes de parenté, les proximités mais par un étrange et fatal signe de connivence, une "marque » commune - la tuberculose. Une anti-société ou une société autre, cosmopolite, venue de tous les horizons européens, nordaméricains et dont la multitude des épitaphes dans toutes les langues et écritures signale la diversité des provenances, des conditions, des fois religieuses. Une société de la maladie qui, lentement ou rapidement, inéluctablement, ronge et conduit à l'issue fatale et qui, poussée par l'espérance d'un répit, d'un sursis, est venue s'échouer sur ce promontoire et mourir au milieu de tant de beauté. Une société dépourvue de toute consistance organique, bien sûr : le poète anglais y côtoie la comtesse russe, l'étudiant allemand, le commerçant tchèque, le diplomate polonais - l'aisance matérielle, condition d'un déplacement et d'une installation aussi coûteux, demeurent l'unique trait d'union entre tous ces morts dont, souvent, la tombe, même en ruines, conserve la trace plus ou moins pompeuse de la condition patricienne. Ces morts-là, venus d'ailleurs, exilés par la phtisie, reposent seuls, contrairement à leurs voisins des caveaux mentonnais, avec leurs assortiments de fleurs en plastique et leurs patronymes à consonance italienne qui, eux, dorment en famille, serrés les uns contre les autres. Mais c'est bien à une sorte d'« anti-ville » des morts que l'on a affaire : une «ville » où les caveaux figurent les maisons, les allées parsemées de graviers les rues, et la petite chapelle orthodoxe construite à la mémoire du Prince Troubetzkoy, un proche du Tsar de toutes les Russies, le lieu de culte destiné à accueillir les fidèles...

7 Plus haut, le « carré du souvenir français » raconte une histoire refoulée, déniée - celle des « indigènes » mobilisés lors de la guerre impérialiste, pour une cause qui leur est 
totalement étrangère. Longue litanie de noms malgaches, souvent écorchés, incomplets (pas de prénom) et qui donne à imaginer le calvaire de ces jeunes hommes arrachés à leur tropique natal pour aller crever de pleurésie dans un hôpital militaire ou encore, l'Armistice signé, succomber à la grippe espagnole sur ce bord de Méditerranée...

8 Tout s'inverse en ce lieu où ce sont les morts, qui, couchés sur leur promontoire observent, « surveillent » de leur œil éteint l'agitation des vivants, en contrebas; où ils se trouvent rassemblés par un "principe » qui défie toutes les règles et taxinomies en vigueur dans les sociétés modernes : le bacille de Kock par opposition à l'appartenance à une même communauté locale ou nationale... Comme lieu, ce cimetière est un espace flottant qui met à mal les logiques territoriales. Il est d'ailleurs situé sur les confins effrangés de l'État-nation français à laquelle Menton n'appartient que depuis 1860, où les habitants du cru continuent de s'exprimer dans un dialecte qui doit davantage à l'italien qu'au français et où, les week-ends, l'italien des visiteurs venus en voisins de Vintimille et ailleurs emplit l'espace sonore de la rue...

On voit bien ici que l'hétérotopie n'est pas seulement un « espace autre », mais aussi un espace voué à la différence, c'est-à-dire à différer d'avec les espaces ordinaires, à faire surgir l'élément d'un différend avec eux : le cliché du "temps arrêté » dans le vieux cimetière de Menton peut retrouver une certaine vigueur si l'on lui assigne une portée critique : le cimetière devient ce lieu où l'on sera moins porté à s'apitoyer sur les "pauvres morts" moissonnés par une maladie qui, sous nos latitudes, a cessé de répandre la terreur et l'effroi (même si elle est loin d'être éradiquée et au contraire continue de faire cortège à la pauvreté et la misère) qu'à s'établir dans une position critique face à ce qui prospère sous nos pieds : le règne de l'argent facile, de la vulgarité des nouveaux riches, un monde d'oisifs amoureux de leurs animaux de compagnie mais sans aménité pour ceux qui les servent - la plèbe innombrable des "personnels de service ». L'hétérotopie devient alors le lieu du retrait non pas blasé ou résigné, mais ironique et lucide. Ce lieu insolite où l'on se sent mieux en compagnie des morts que des vivants. Mystérieusement « en communion » avec ces morts, d'autant plus que l'on s'éprouve étranger au monde clinquant qui s'expose en contrebas.

10 Je veux dire par là que le mort, « habitant » de cette ville hétérotopique, le cimetière, devient le compagnon idéal, idéal et paradoxal, d'une muette méditation, d'une muette protestation contre les formes contemporaines de la vie, contre la laideur de cette modernité là, dans cette topographie-là - celle de cette Côte d'Azur-là, monde de maîtres et de serviteurs, de riches et de pauvres jusqu'à la caricature, monde « romain » en somme auquel ne manquent, mais de peu, que les esclaves et les combats de gladiateurs. "La vie », étrangement, semble s'y déplacer du côté des morts tant la futilité y trame le présent de ce monde sans épaisseur, y arrête le temps dans une sorte d'artifice du loisir perpétuel - et vide.

11 Car qu'est-ce qu'un mort, au fond ? C'est une personne humaine qui est dissociée de son corps. Une personne humaine dont les formes de "vie", ultérieures à son décès, peuvent être innombrables - on ne meurt jamais tout à fait, pour autant qu'on appartient à une communauté -, mais en tout cas se produisent dans l'élément de, ou à la condition de cette dissociation de la personne d'avec son corps. Un mort, c'est donc, selon une tradition philosophique et religieuse immémoriale, aux multiples facettes, une personne émancipée de son corps. Il y a donc un très fort paradoxe à venir faire communauté avec les morts, à venir philosopher avec eux sur les mauvais plis du contemporain, de l'époque - là où précisément sont conservés ces "restes" qui 
constituent le plus inessentiel dans la vie d'un mort; là, où, comme dit Foucault, un espace est assigné à un corps - celui du tombeau. Pour Foucault, en effet, ce sont «le miroir et le cadavre qui assignent un espace à l'expérience profondément et originairement utopique du corps ", c'est-à-dire l'astreignent à une condition d'unicité topologique - en lui donnant son lieu, unique et "sans recours». Pour le reste, l'expérience du corps est, chez les humains, intrinsèquement utopique : elle est celle du corps qui se transforme, se diffracte, se dissocie, du corps qui, littéralement n'a pas de lieu. Je cite : «Mon corps, c'est comme la Cité du Soleil, il n'a pas de lieu, mais c'est de lui que sortent et que rayonnent tous les lieux possibles, réels ou utopiques ».

L'accent porté par Foucault sur la condition utopique du corps nous permet de découvrir, de dévoiler ce qui constitue le faux-semblant de tout cimetière, tout particulièrement, ici, du vieux cimetière de Menton: ce qui fait d'un cimetière une hétérotopie ou, plutôt, ce qui lui permet d'être, en puissance, une hétérotopie, c'est qu'il n'est pas, en réalité, et contrairement aux apparences, un dépôt de cadavres, de dépouilles humaines. Les corps n'y reposent pas, n'y sont pas conservés, ils y disparaissent, s'y dispersent, en retournant à la terre, pour laisser la place aux morts dont la présence se manifeste par la permanence de leurs noms propres, leur persistance bien après que les corps se soient dispersés. Un cimetière est donc bien un lieu habité par des morts et non pas peuplé de cadavres, que l'on peut venir côtoyer, auxquels les proches rendent visite, à la Toussaint ou tous les jours - il y en a même auxquels ceux qui souffrent sans fin de leur perte viennent parler, confier leurs douleurs et leurs secrets.

13 Et donc, au vieux cimetière de Menton, ce qui va me porter à faire communauté avec ces morts, à partager avec eux mon aversion de ce qui se joue un peu plus bas dans la petite ville si douce, si attachante encore au temps de mon enfance et désormais profanée par le tourisme processionnaire et l'horreur ploutocratique, c'est ce que j'appellerai le partage de la désinscription, de l'exil, du déracinement : venus de tous les horizons, de toutes les langues, de toutes les conditions, de toutes les croyances auxquels les a arrachés un fléau ou un autre - la tuberculose, la guerre mondiale -, opulents commerçants d'Europe centrale, dame de compagnie de l'impératrice russe ou aussi bien pauvres conducteurs de troupeaux des plateaux malgaches, bergers de l'Atlas marocain - tous me sont proches, dans leur condition d'acosmiques, d'expatriés venus reposer sur le promontoire de cet autre cimetière marin d'où, par temps clair, se distingue la silhouette noire et escarpée de la Corse, au loin... Oui, avec eux, je peux partager le secret de la rancune tenace que m'inspire le désastre de ce lieu qui a enchanté mon enfance, et aussi, pourquoi le cacher, l'angoisse que m'inspire la disparition annoncée, en ces lieux, de mes parents.

Or ce lieu, le vieux cimetière de Menton, m'est précieux, ces morts mes compagnons me sont précieux, pour la raison même que dit Foucault, dans un autre texte intitulé « Les hétérotopies »: "On ne vit pas dans un espace neutre et blanc; on ne vit pas, on ne meurt pas, on n'aime pas dans le rectangle d'une feuille de papier. On vit, on meurt, on aime dans un espace quadrillé, découpé, bariolé, avec des zones claires et sombres, des différences de niveaux, des marches d'escalier, des creux, des bosses, des régions dures et d'autres friables, pénétrables, poreuses ». On vit, donc, dans des espaces différenciés, variablement investis, susceptibles donc de s'hétérogénéiser. Foucault, encore : "Or, parmi ces lieux qui se distinguent les uns des autres, il y en a qui sont absolument différents : des lieux qui s'opposent à tous les autres, qui sont destinés en quelque sorte 
à les effacer, à les neutraliser ou à les purifier. Ce sont en quelque sorte des contreespaces ». Et, ajoute encore Foucault, renouant avec le motif de l'enfance que j'ai mobilisé plus haut, les enfants, tout particulièrement, « les connaissent bien », ces lieux et font de leur investissement un mode de vie - le jardin, le grenier, la tente d'Indien, le grand lit des parents, etc. Thématique benjaminienne, s'il en fût.

En nous déplaçant du côté de l'hétérotopie - un tout petit pas y suffit parfois, mais un long voyage n'y pourvoira pas nécessairement -, nous nous mettons en position de différer d'avec le présent. La raison pour laquelle les cimetières ont, dans nos sociétés, une forte charge hétérotopique est bien évidente - la différence du mort d'avec le vif n'est-elle pas le paradigme de toute différence, en un sens non pas relatif mais absolu? En ce sens, l'omniprésence des cimetières (un phénomène qui nous est si familier que nous ne le remarquons même plus) dans nos villes rend manifeste l'évidence de cette inscription d'une véritable pulsion hétérotopique au cœur de notre condition: plus rigoureusement nous sommes "territorialisés ", assignés à une multitude de tâches, de fonctions, de définitions, de normes, et plus est impérieux notre désir d'échappée vers ces « espaces autres». Tout, dans ces conditions, est susceptible de se voir doté d'un devenir hétérotopique, à la mesure même où rien n'est assuré de persévérer dans un être hétérotopique intrinsèque... De ce point de vue, je serais porté à insister davantage que ne le fait Foucault sur le devenir hétérotopique, davantage que sur la condition hétérotopique. Envahi par des hordes de touristes bruyants et filmeurs compulsifs, ce qui doit être le cas autour du 15 août, le vieux cimetière de Menton cesse d'être une hétérotopie, tout comme le carnaval, événement hétérotopique par excellence, dès l'instant où, comme celui de Nice, il se transforme en business géré par le comité des fêtes local... Inversement, tout ou à peu près tout peut devenir une hétérotopie, à condition d'être saisi par un processus de différenciation au fil duquel l'unité du territoire habité va se briser, la continuité va faire place à des jeux d'opposition entre le familier et l'étrange, le partagé et le secret, l'ordinaire et l'extraordinaire, etc. De ce point de vue, on pourrait dire tout simplement qu'il y a des hétérotopies, que de nouvelles hétérotopies apparaissent sans cesse au fur et à mesure que d'autres disparaissent en se «territorialisant »- tout simplement parce que le vivant est porté à s'hétérogénéiser, à varier, à se singulariser en différant, etc. - Canguilhem, entre autres.

Pas d'hétérotopies, de ce point de vue, sans processus de subjectivation - chacun a ses hétérotopies "personnelles» plus ou moins parfaites et qui le seront moins pour d'autres - ou pas du tout... Chacun a son petit «trésor » personnel d'hétérotopies chacun pouvant être un sujet individuel ou un groupe de dimension variable.

Exemples - personnels, donc :

- La foire du livre de Ruynes en Margeride, dans le Cantal, tous les 14 juillet : un rite, je m'en réjouis à l'avance, le trajet (on n'entre pas dans une hétérotopie comme dans un moulin, rappelle Foucault), un « autre » 14 juillet (sans bal ni fanfare) la quête du livre rare, de l'occasion providentielle qui, à son tour, va susciter une bifurcation hétérotopique, détourner des programmes de lecture soigneusement fixés à l'avance... Mais aussi l'occasion d'un partage avec les amis, d'une initiation... Une hétérotopie éphémère, mais récurrente... On se désole quand on ne peut s'y rendre, pour cause de maladie, de déplacement à l'étranger..

- Un village aborigène depuis longtemps réabsorbé par la forêt, sur les traces infimes duquel nous conduisent les derniers des Bunun (un peuple « premier » de l'île, dans la montagne de 
Taiwan). La marche de plusieurs heures qui y conduit, éprouvante, inquiétante, prend une allure de parcours initiatique. Et lorsqu'on y parvient - rien ou presque, si ce n'est l'émotion perceptible de nos guides : ici ont vécu les leurs, il y avait même une église, une école - avant que l'armée japonaise ne les en chassent afin de les regrouper, tactique familière de tous les colonisateurs, dans des « villages » sous contrôle. Chasseurs acharnés aujourd'hui encore, nos amis Bunun ont inscrit ce lieu secret sur leurs parcours invisibles. L'âme de leurs ancêtres l'habite, quand bien même nous n'y verrions qu'un entrelacs de buissons et d'arbres centenaires.

- Tout bon cinéma, tout bon film cultivent silencieusement leurs hétérotopies, agence sur elle ses lignes de fuite. Ainsi, dans La question humaine de Nicolas Klotz et Elisabeth Perceval, film au sujet particulièrement lourd et grave - de la « rationalisation » de l'organisation $\mathrm{du}$ travail dans l'entreprise aujourd'hui à la production industrielle des cadavres. Mais voici que, sans qu'aucune nécessité narrative impérieuse l'impose, surgit le motif hétérotopique une cave vouée au grand art du flamenco, un bar où officient des filles libres et rieuses, une île improbable où s'improvise une rave-party, un petit restaurant ouvrier de province où l'on aimerait avoir ses habitudes... autant de lieux autres et qui nous suggèrent : non, la boucle n'est pas bouclée, notre histoire, passé, présent, futur n'est pas totalement enfermée dans le sombre paradigme que construit le film...

Bref: un ailleurs est toujours possible. Dans la découverte, la pratique d'une hétérotopie, nous éprouvons notre capacité, notre puissance de différer d'avec nousmêmes - et le monde. Foucault, ici, avec ce motif apparemment mineur dans son travail, « croise » Deleuze une fois encore, non sans réveiller Nietzsche - rien de moins !

\section{BIBLIOGRAPHIE}

Michel Foucault, Le corps utopique, les hétérotopies, avec une présentation de Daniel Defert, Lignes, 2009.

\section{RÉSUMÉS}

Le cimetière, ou plutôt $\mathrm{CE}$ cimetière, en tant que singularité, est envisagé dans sa fonction hétérotopique : en tant que point d'inversion des normes et des conduites et dans sa condition excentrée du point de vue des logiques du territoire. Ce trait spécifique dote ce lieu ou espace « autre » d'une capacité objectivement critique - lieu de retrait, de surplomb, d'excentrement à la fois proche et lointain dans son rapport à la «vie courante». Sous l'angle hétérotopique, le mort saisit le vif par des biais tout à fait insolites - les puissances du mort (plutôt que de la mort) ne sont ici nullement funèbres ou funéraires, et si elles ont part liée avec une certaine forme de nostalgie, celle-ci tend à prendre un tour politique - le déplacement hétérotopique OUVRE LES YEUX sur le topique ordinaire... 
INDEX

Mots-clés : cimetière, hétérotopie, Menton, mort

AUTEUR

ALAIN BROSSAT

Professeur université Paris VIII 